\title{
Smartphone: usos y gratificaciones de los jóvenes en México en 2015
}

\section{María Elena Gutiérrez-Rentería ${ }^{1}$ Josefina C. Santana-Villegas ${ }^{2}$ Martha Pérez-Ayala ${ }^{3}$}

Recibido: 2015-11-14

Enviado a pares: 2015-11-23
Aprobado por pares: 2016-01-26

Aceptado: 2016-02-01

DOI: 10.5294/pacla.2017.20.1.3

Para citar este artículo / to reference this article / para citar este artigo

Gutiérrez-Rentería, M. E., Santana-Villegas, J. C., y Pérez-Ayala M. (2017). Smartphone: usos y gratificaciones de los jóvenes en México en 2015. Palabra Clave, 20(1), 47-68. DOI: $10.5294 /$ pacla.2017.20.1.3

\section{Resumen}

Esta investigación forma parte de un estudio realizado entre Chile, los Estados Unidos y México para identificar los usos que los jóvenes hispanos les dan a sus teléfonos inteligentes conocidos como smartphones. El objetivo del estudio es identificar los hábitos de consumo, las preferencias de este medio respecto de otros, así como las principales actividades que realizan los jóvenes mexicanos a través de su teléfono celular. El método de investigación que guía el estudio es mixto, dado que se utilizan técnicas para obtener los resultados de forma cualitativa, a través de encuestas focales, y cuantitativa, a través de encuestas en línea. La población indagada forma parte de los jóvenes de entre 18 y 25 años que estudian en una Universidad privada en el área metropolitana de Guadalajara. En las encuestas focales participaron 27 alumnos y se aplicaron 707 encuestas en línea. Los resultados muestran que el smartphone ha puesto al alcance aplicaciones 
que satisfacen diversas necesidades de comunicación de forma instantánea; los jóvenes utilizan este dispositivo más de cinco horas diarias, siendo WhatsApp la red social más importante para comunicarse con su familia y amigos. A manera de conclusión, puede decirse que, para esta población estudiada, existe una relación directa respecto del género de la persona para la elección de la marca, el tiempo de consumo y, finalmente, para la elección de uso de los smartphones frente a otras redes sociales en línea y medios de comunicación.

\section{Palabras clave}

Celular; jóvenes; redes sociales; México (Fuente: Tesauro de la Unesco). 


\section{Smartphone: Uses and Rewards for Mexican Youth in 2015}

\section{Abstract}

This research is part of a study conducted between Chile, the United States and Mexico to identify how Hispanic young men and women use their smartphones. The purpose of the study is to identify the consumption habits, the preferences of this means as compared to others, as well as the main activities performed by young men and women in Mexico on their cell phones. The research method used in the study is a mixed method, as it uses techniques to obtain the results in a qualitative (through focal surveys) and quantitative (through online surveys) manner. The researched population is made by a group of young men and women from 18 to 25 years of age, currently enrolled at a private university from the metropolitan area of Guadalajara. Twenty-seven students participated in the focal surveys and 707 online surveys were also conducted. Results show that smartphones have made available certain applications that instantly meet the different communication needs; youngsters use these devices for over five hours a day, WhatsApp being the most important social network to communicate with family and friends. In conclusion, you could say that, according to the population studied, the gender of the person is directly related to choosing the brand, time of consumption and, finally, choosing how to use the smartphones as compared to other online social networks and means of communication.

\section{Keywords}

Cell Phone; Youth; Social Networks; Mexico (Source: Unesco Thesaurus). 


\section{Smartphone: usos e gratificações dos jovens no México em 2015}

\section{Resumo}

Esta pesquisa forma parte de um estudo realizado entre Chile, os Estados Unidos e o México para identificar os usos dados pelos jovens hispanos aos seus telefones inteligentes conhecidos como smartphones. $\mathrm{O}$ objetivo do estudo é identificar os hábitos de consumo, as preferências deste meio com respeito a outros, assim como as principais atividades que os jovens mexicanos realizam através de seu telefone celular. $\mathrm{O}$ método de pesquisa que guia o estudo é misto, dado que se utilizam técnicas para obter os resultados de forma qualitativa, através de enquetes focais, e quantitativa, através de enquetes online. A população indagada forma parte dos jovens entre 18 e 25 anos que estudam em uma universidade privada na área metropolitana de Guadalajara. Nas enquetes focais participaram 27 alunos e foram realizadas 707 enquetes online. Os resultados mostram que o smartphone pôs ao alcance aplicações que satisfazem diversas necessidades de comunicação de forma instantânea; os jovens utilizam este dispositivo mais de cinco horas diárias, sendo o WhatsApp a rede social mais importante para comunicar-se com sua família e seus amigos. Como conclusão, pode-se dizer que, para esta população estudada, existe una relação direta no que se refere ao gênero da pessoa para a escolha da marca, o tempo de consumo e, finalmente, para a escolha de uso dos smartphones perante as outras redes sociais online e a mídia.

\section{Palavras-chave}

Celular; jovens; redes sociais; México (Fonte: Tesauro da Unesco). 


\section{Introducción}

La forma en que se comunican los jóvenes y el uso que le dan al teléfono celular con acceso a internet, llamados teléfono inteligente o smartphone, son quizá algunos de los temas de mayor interés en el ámbito económico, académico y social. Entre ciertos sectores de la población en México, es difícil encontrar a alguien que no tenga un dispositivo móvil con acceso a internet. La baja en los precios en la industria de las telecomunicaciones y los diferentes paquetes ofrecidos por las empresas de telefonía celular han contribuido a que este medio sea de fácil acceso para cualquier ciudadano en este país.

Este estudio tiene como objetivo identificar los principales usos y gratificaciones que los jóvenes universitarios en México, entre 18 y 25 años, le dan al Smartphone, en 2015. Esta investigación forma parte de un estudio que siguió la misma metodología en los Estados Unidos, Chile y México. El origen del trabajo es en 2008 gracias a la invitación realizada por Alan Albarran, de la North Texas University, que hizo a un grupo de investigadores en América Latina, cuyos resultados fueron publicados por el mismo autor en 2009 (Albarran, 2009), así como por otros investigadores en diversas revistas científicas (Van-Weezel y Benavides, 2009).

La estructura de esta investigación está dividida en cuatro partes. La primera sección trata sobre los antecedentes de la industria de las telecomunicaciones en México. La segunda estudia las principales aportaciones académicas que se han desarrollado sobre el objeto de estudio, principalmente en México. La tercera parte profundiza en la metodología utilizada en el estudio, y en el cuarto apartado se presentan los resultados.

\section{Antecedentes de la industria}

Las tecnologías de comunicación digital en México forman parte de la vida cotidiana de la mayoría de la población de este país. El $42 \%$ de los hogares en México tiene telefonía fija en sus hogares, mientras que $80 \%$ de estos hogares cuenta con al menos un teléfono móvil y cerca de $35 \%$ tiene al menos una computadora (Inegi, 2014). Según el Instituto Nacional de 
Estadística y Geografía (Inegi), al menos 9 de cada 10 hogares tiene un teléfono celular y la introducción de internet es más de $30 \%$.

Respecto del perfil de los internautas en México, el Inegi señala que en 2013 cerca de $47 \%$ de la población es usuaria de computadora, y la que utiliza internet llega a casi $44 \%$, mientras que el celular es de uso para más de la mitad de los habitantes. Por otra parte, la población conectada a internet en México alcanzó $51 \%$ de las personas mayores de 6 años, que significa 54 millones de usuarios (Ampici, 2015). El segmento de edad en el que se concentra el mayor número de internautas está compuesto por personas que tienen entre 19 y 34 años, y el número de horas promedio que el mexicano usa internet es de aproximadamente 6 horas.

Por otro lado, ComScore (2015) afirma que las principales actividades en internet son destinadas al uso de las redes sociales, el entretenimiento y a visitar diversos portales a través de los dispositivos móviles, entre ellos, el smartphone y los famosos gadgets. La población internauta dedica la mayor parte de su tiempo a las redes sociales, a contenidos de entretenimiento $y$ un bajo porcentaje al acceso de información y noticias.

\section{Literatura}

Han sido diversos los estudios realizados sobre el uso que hacen los jóvenes respecto de las tecnologías digitales, en concreto hacia los teléfonos móviles conocidos como smartphones, y sobre el uso de las redes sociales en internet. Los objetivos de estas investigaciones varían según el país de procedencia en el que se desarrolla el estudio, pero, principalmente, las preguntas sobre este fenómeno social se elaboran desde la perspectiva educativa, del conocimiento del mercado y del fenómeno de la comunicación (Katz, Blumler y Gurevitch, 1973; Ruggiero, 2000; Campbell, 2007; Van-Weezel y Benavides, 2009; Joo y Sang, 2013; García-de-Torres et al., 2011).

Qiaolei y Yi-Hsuan (2015) identifican los síntomas de dependencia y sociabilidad de jóvenes universitarios en Singapur. Otro estudio similar realizado por Darcin et al. (2015) muestra el alto nivel de adicción en jóvenes turcos que tuvieron smartphones en sus primeros años y aquellos que 
utilizan principalmente sus teléfonos inteligentes para acceder a las redes sociales en la red.

Por otra parte, Elizondo, Bernal y Montoya (2010) analizan los recursos de aprendizaje móvil de jóvenes universitarios mexicanos, y concluyen que el uso de los recursos m-learning (mobile learning) modifica el ambiente de aprendizaje al convertir el escenario en un ambiente innovador y colaborativo, necesario para las generaciones interactivas. Otro estudio realizado en México relacionado con el uso de los dispositivos móviles en los jóvenes es el de Herrera (2009), en el que se destaca la alta popularidad de Google y Wikipedia, el uso de Messenger para socializar y las redes sociales Myspace, hi5 y Facebook. Respecto del medio digital, el teléfono móvil era el más significativo para los estudiantes. Finalmente, concluye que 88 \% de los estudiantes no había utilizado la infraestructura tecnológica instalada de las universidades dedicadas a las plataformas educativas.

Respecto de otras investigaciones relacionadas con el tema de las redes sociales en México, está la de Domínguez y López (2015), quienes hacen un análisis sobre las investigaciones realizadas en México, y concluyen que los estudios son pocos sobre el uso que hacen los jóvenes de sus teléfonos celulares y redes sociales en internet.

En 2008 Albarran (2009), junto con un grupo de investigadores latinoamericanos, llevó a cabo el estudio "Young latinos use of mobile phones: a cross-cultural study", que tuvo como marco teórico los usos y las gratificaciones, dado que este método ayuda a mostrar cómo la audiencia o el consumidor es selecto respecto del consumo de información y entretenimiento, así como de las tecnologías que satisfacen sus necesidades y deseos. La metodología utilizada en esa investigación fue cualitativa y cuantitativa para identificar el uso que los jóvenes hispanos hacían de sus teléfonos inteligentes, además de que se compararon los beneficios obtenidos de su uso frente a otros medios de comunicación. Los países representados en el estudio fueron la Argentina, Chile, Colombia, México y el Uruguay. Finalmente, este autor desarrolló otra investigación respecto del uso que hacen los jóvenes hispanos de las redes sociales en internet (Albarran, 2013). 


\section{Metodología}

El trabajo que aquí se presenta tiene como principal objetivo profundizar en el uso que le dan los jóvenes mexicanos a sus teléfonos celulares, en cómo se comunican con su familia y amigos a través de este dispositivo electrónico. También pretende identificar el comportamiento de los jóvenes a través de su teléfono para el consumo de información y entretenimiento y conocer en qué medida los jóvenes mexicanos prefieren el smartphone respecto de otros medios tradicionales de comunicación. Por último, también es oportuno descubrir si influye el género de la persona y los niveles de ingreso en las preferencias de uso.

El método de investigación que guía el estudio es mixto, dado que se utilizan técnicas para obtener los resultados de forma cualitativa, a través de encuestas focales, y cuantitativa, a través de encuestas en línea. Tanto la guía de entrevista en las encuestas focales como las preguntas de los cuestionarios fueron las mismas utilizadas en la investigación intercontinental realizada con los Estados Unidos y Chile en 2015. Es importante decir que fueron 20 las preguntas formuladas para las entrevistas focales y 26 las utilizadas en el cuestionario.

Todos los participantes son estudiantes de la misma Universidad privada en la zona metropolitana de Guadalajara. Los alumnos que participaron en el estudio fueron convocados por los coordinadores de estudio. En los grupos focales, participaron 27 estudiantes de la Escuela de Comunicación el 24 de marzo de 2015. Los cuatro grupos focales estuvieron formados por 14 mujeres y 13 varones. Las entrevistas fueron grabadas en audio $\mathrm{y}$ video, $\mathrm{y}$ transcritas.

En el estudio cuantitativo, que son las encuestas en línea, se utilizó una probabilidad de acierto ( $\mathrm{p}$ ) de 0.5 para determinar el tamaño de la muestra. El nivel de confianza utilizado fue de $95 \%$ y un error de estimación de $3.74 \%$. Esto da un tamaño de la muestra de 707 alumnos que fueron las encuestas aplicadas en la comunidad universitaria. Las encuestas fueron distribuidas por los coordinadores académicos. Se recibió respuesta de estudiantes de las licenciaturas de Educación, Comunicación, Ingeniería, así 
como de la Escuela de Economía, Administración y Negocios. El periodo en el que se realizaron las encuestas es del 23 de marzo al 15 de septiembre. La edad promedio de los encuestados es de 20 años.

\section{Resultados}

\section{Fase cualitativa: encuestas focales}

Los resultados de las encuestas focales muestran que estos jóvenes utilizan el smartphone en más de la mitad del tiempo de actividad durante el transcurso del día. En promedio, los alumnos señalaron dedicar cerca de 6.7 horas diarias al uso de este durante un típico día entre semana y cerca de 7.3 horas durante un día típico el fin de semana.

Por otra parte, $100 \%$ de los entrevistados consideraron el smartphone como "una herramienta indispensable en la vida cotidiana, que favorece la comunicación, el entretenimiento y la actualización de la información de forma instantánea". Sin embargo, $40 \%$ de los estudiantes universitarios considera que no es relevante la mayor parte de la información que recibe diariamente.

Los mensajes de texto a través de WhatsApp son el medio de mayor comunicación para los jóvenes. El 87 \% de la población participante en los grupos focales utiliza esta red social. Por otra parte, $71 \%$ de los jóvenes prefiere externar y dialogar en persona o por teléfono. Dos expresiones realizadas por dos alumnos que sirven para identificar la valoración afectiva que tienen al hacer uso de la aplicación WhatsApp o los mensajes de texto. Para estos alumnos, esta herramienta de comunicación sirve para expresar sentimientos cuando "es complicado decirlo cara a cara" o cuando existen momentos en que "no tienes ganas de hablar con nadie y prefieres escribirlo".

El $93 \%$ de los jóvenes utiliza el WhatsApp para compartir información y comunicarse con sus amigos. El 67 \% de esta misma población utiliza Instagram. Otras redes sociales que también son utilizadas, pero con menor frecuencia e intensidad, son Snapshot, con tan solo $27 \%$, así como Facebook y Spotify. Estas últimas solo son usadas por $20 \%$ de los alumnos. 
Finalmente, $20 \%$ de los jóvenes considera las redes sociales como opción para hacer amigos, siempre y cuando sean amistades de otras amistades, exista la posibilidad de interacción con una persona que se conoció personalmente o se considere que puede darse un mayor afecto en una relación de amistad o amorosa. Aunque 13 \% de la misma población estima que las redes sociales no son opción para hacer nuevos amigos.

En cuanto a las preferencias del smartphone, respecto de otros medios de comunicación tradicional, $100 \%$ de los participantes afirmó tener aplicaciones que los han sustituido, principalmente, el periódico impreso. El $66 \%$ de la población afirma utilizar los medios informativos digitales para estar enterados de las noticias y para fines escolares. Algunas de las empresas mencionadas por los alumnos como fuente de información a través de su teléfono móvil son CNN, Mural (Grupo Reforma) y El Informador.

Existe poco uso de la radio en el celular por parte de estos jóvenes. El $100 \%$ de los participantes señaló que las aplicaciones iTube y YouTube han sustituido el uso de la radio comercial abierta para escuchar música. Por otra parte, solo $7 \%$ de la población tiene instalada una aplicación de revista. El $100 \%$ de los participantes mencionó utilizar la aplicación de televisión para conectarse directamente a las pantallas inteligentes, o televisiones inteligentes, conocidas como las Smart TV.

Todos los jóvenes participantes definen el teléfono celular como la herramienta más práctica y accesible, debido al tamaño, el acceso a la comunicación y la música. Finalmente, $100 \%$ de esta población rechaza recibir publicidad a través de tal dispositivo de comunicación.

\section{Resultados de la fase cuantitativa: encuestas}

1) El $98 \%$ de los encuestados tiene internet en su teléfono celular, así como la mayoría de las aplicaciones propias de un teléfono inteligente. El porcentaje menor de las aplicaciones instaladas en el teléfono celular es el de juegos (tabla 1).

Más de $90 \%$ de los encuestados reconoció tener todas las once aplicaciones en sus teléfonos inteligentes. Excepto el MP3 player y los juegos. 


\section{Tabla 1. Aplicaciones que tienen los smartphones}

\begin{tabular}{|c|l|c|}
\hline & Tipo de aplicaciones & $\%$ \\
\hline 1 & Mensajes de texto & 95 \\
\hline 2 & Acceso a internet & 98 \\
\hline 3 & MP3 player & 83 \\
\hline 4 & Cámara de fotos & 98 \\
\hline 5 & Cámara de video & 93 \\
\hline 6 & Calendario & 93 \\
\hline 7 & Reloj/alarma & 97 \\
\hline 8 & Juegos & 73 \\
\hline 9 & Acceso al correo electrónico & 95 \\
\hline 10 & Notas & 92 \\
\hline 11 & GPS & 93 \\
\hline
\end{tabular}

Muestra 707 encuestados.

Las mujeres reconocen tener mayor porcentaje en el uso del MP3 player y en juegos, comparados a los varones.

2) Más de $70 \%$ de los jóvenes tiene contrato anual con una empresa de telefonía celular; más de $20 \%$ accede al servicio a través del prepago y cerca de $5 \%$ tiene incluido el servicio a través de paquetes que incluyen telefonía fija, televisión de pago e internet. Por otra parte, existe una amplia relación entre el tipo de contrato que tienen con la compañía celular y el nivel de ingresos de las personas. En la medida en que se incrementa el ingreso, aumenta el porcentaje de personas con contratos anuales y disminuyen los contratos de prepago.

3) Las tres marcas más usadas son Apple, Samsung y Sony. Siendo Apple la marca más utilizada y que acapara más de $65 \%$ del total de los alumnos encuestados. La figura 1 muestra las principales marcas utilizadas por esta población en 2015.

Por otra parte, existe una correlación directa entre las marcas de teléfonos inteligentes que usan los universitarios y los niveles de ingresos que reportaron. Las cinco marcas más usadas son: Apple (62.10\%), Samsung 


\section{Figura 1. Marcas de smartphones utilizadas}

por los jóvenes mexicanos

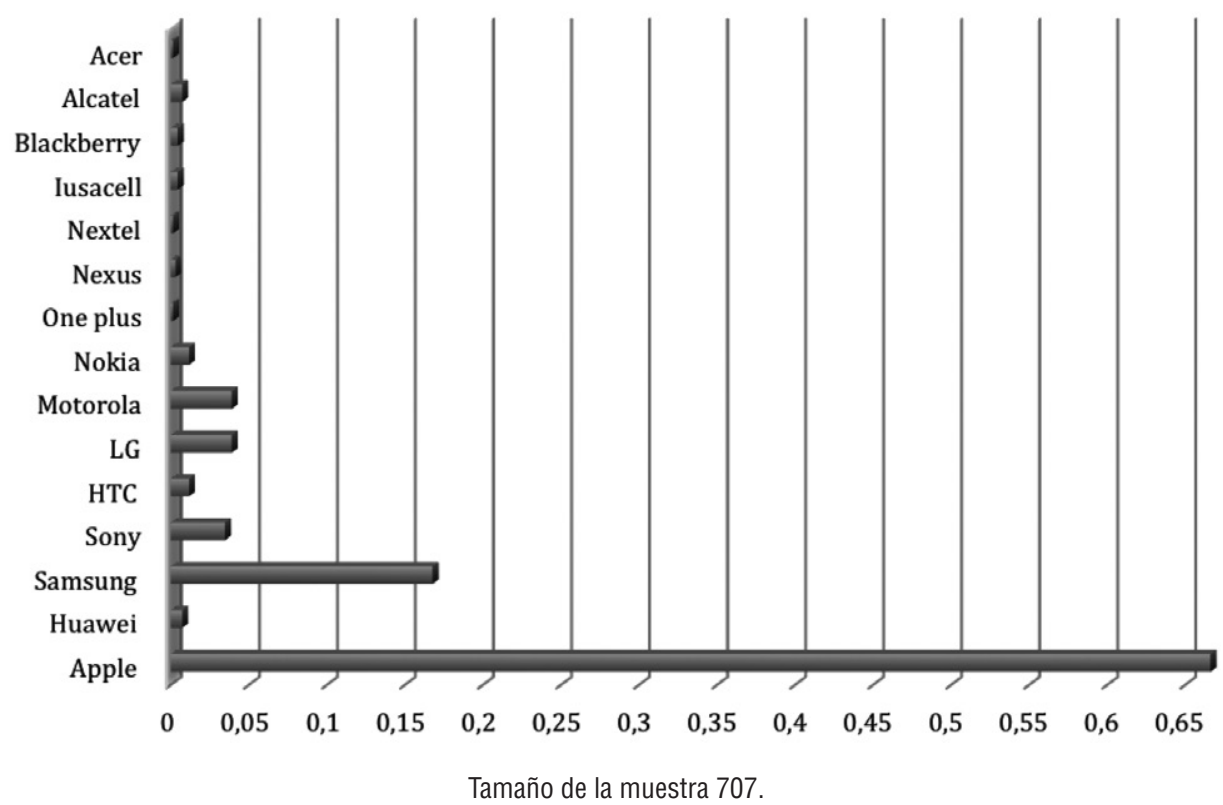

(18.55 \%), LG (4.65 \%), Motorola (4.65 \%) y Sony (3.23\%). Estas cinco marcas concentran más de $90 \%$ de las usadas por los jóvenes.

A mayor nivel de ingresos, mayor cantidad de alumnos utilizan Apple, Sony y Motorola. Sin embargo, Samsung se mantiene constante a lo largo de todos los niveles socioeconómicos. Y las marcas LG se concentran en los niveles de ingresos más bajos. Asimismo, existe preferencia hacia la marca según género de la persona. El $60.47 \%$ de las mujeres tiene la marca Apple; y $49.28 \%$, los varones. Por otra parte, los hombres registran tener $26.81 \%$ de la marca Samsung, mientras que las mujeres tan solo $16.28 \%$. Otro ejemplo es la marca Sony, que los varones registran tener $6.52 \%$; y $2.33 \%$, las mujeres (tabla 2).

4) Los jóvenes dedican una hora promedio en un día típico entre semana para hablar (audio) por teléfono a través de su teléfono inteligente, poco más de 10 horas en promedio durante un día el fin de semana y poco 
Tabla 2. Uso de marca de smartphones respecto del género

\begin{tabular}{|c|c|c|c|c|}
\hline \multirow{2}{*}{} & \multicolumn{2}{|c|}{ Total de la muestra } & \multicolumn{2}{c|}{ Género } \\
\cline { 2 - 5 } & Frec & $\%$ & $\%$ & Femenino \\
\hline Apple & 308 & 62.10 & 49.28 & 60.47 \\
\hline Blackberry & 3 & 0.60 & $2.17 \%$ & 0.00 \\
\hline Galaxy & 1 & 0.20 & 0.00 & 0.00 \\
\hline Iusacell & 3 & 0.60 & 0.72 & 0.00 \\
\hline LG & 23 & 4.64 & 2.90 & 11.63 \\
\hline Motorola & 23 & 4.64 & 5.07 & 6.98 \\
\hline Nextel & 1 & 0.20 & 0.72 & 0.00 \\
\hline Nexus & 2 & 0.40 & 0.00 & 0.00 \\
\hline Nokia & 4 & 0.80 & 2.17 & 0.00 \\
\hline Samsung & 92 & 18.55 & 26.81 & 16.28 \\
\hline Sony & 16 & 3.23 & 6.52 & 2.33 \\
\hline Alcatel & 5 & 1.01 & 0.00 & 2.33 \\
\hline Huawei & 1 & 0.20 & 0.00 & 0.00 \\
\hline HTC & 4 & 0.80 & 1.45 & 0.00 \\
\hline Otras & 10 & 2.02 & 2.17 & 0.00 \\
\hline
\end{tabular}

i 2 con 28 grados de libertad $=470371$, $p$-value $=0.0136$.

Tamaño de la muestra 707. Nivel de confianza de $95 \%$.

más de 11 horas durante un típico día en las vacaciones. Por otro lado, el tiempo dedicado a mandar o recibir mensaje de texto aumenta considerablemente. Los jóvenes dedican 1.3 horas en promedio durante un típico día entre semana para recibir o mandar textos a través de su smartphone; 6.3 horas promedio durante un típico día durante el fin de semana, y 7.7 horas en promedio durante un típico día durante las vacaciones.

No existe diferencia entre las medias de hombres y mujeres respecto de hablar por teléfono a través de su celular entre semana. Sin embargo, durante los fines de semana y vacaciones sí existe una significativa diferencia en el tiempo dedicado al medio y al género de la persona. Por otra parte, también existe diferencia notable entre hombres y mujeres en cuanto al uso que le dan al celular para mandar y recibir mensajes de texto. Las mujeres tienden a tener promedios más altos en los tiempos que dedican a estas actividades que los hombres. Puede afirmarse que las mujeres utilizan 
más el audio y dedican mayor tiempo a mandar y recibir correos durante los fines de semana y las vacaciones (tabla 3 ).

\section{Tabla 3. Diferencias de medias entre géneros respecto del tiempo que utilizan los jóvenes su smartphone para hablar o mandar y recibir texto}

\begin{tabular}{|c|c|c|c|}
\hline \multirow{2}{*}{} & \multicolumn{2}{|c|}{ Género } & \\
\cline { 2 - 4 } & Masculino & Femenino & F-Snedecor \\
\hline $\begin{array}{c}\text { ¿Cuántas horas en un día típico gastas en hablar } \\
\text { a través de tu teléfono celular? Típico día entre } \\
\text { semana. }\end{array}$ & $\begin{array}{c}\text { Media }=1 \\
\text { Muestra }=138\end{array}$ & $\begin{array}{c}\text { Media }=1 \\
\text { Muestra }=315\end{array}$ & $\begin{array}{c}\mathrm{F}(2,493)=0 \\
(\mathrm{p}=1.0000)\end{array}$ \\
\hline $\begin{array}{c}\text { ¿Cuántas horas en un día típico gastas en hablar a } \\
\text { través de tu teléfono celular? Típico día durante el } \\
\text { fin de semana. }\end{array}$ & $\begin{array}{c}\text { Media }=8.7803 \\
\text { Muestra }=138\end{array}$ & $\begin{array}{c}\text { Media }=11.7607 \\
\text { Muestra }=138\end{array}$ & $\begin{array}{c}\mathrm{F}(2,492)=83.2959 \\
(\mathrm{p}=0.0000)\end{array}$ \\
\hline $\begin{array}{c}\text { ¿Cuántas horas en un día típico gastas en hablar a } \\
\text { través de tu teléfono celular? Típico día durante las } \\
\text { vacaciones. }\end{array}$ & $\begin{array}{c}\text { Media }=10.1098 \\
\text { Muestra }=138\end{array}$ & $\begin{array}{c}\text { Media }=12.3324 \\
\text { Muestra }=138\end{array}$ & $\begin{array}{c}\mathrm{F}(2,493)=89.473 \\
(\mathrm{p}=0.0000)\end{array}$ \\
\hline $\begin{array}{c}\text { ¿Cuántas horas gastas mandando o leyendo } \\
\text { mensajes en un día? (incluye SMS, WhatsApp, } \\
\text { Facebook). Típico día entre semana. }\end{array}$ & $\begin{array}{c}\text { Media }=1 \\
\text { Muestra }=138\end{array}$ & $\begin{array}{c}\text { Media }=1.6222 \\
\text { Muestra }=138\end{array}$ & $\begin{array}{c}\mathrm{F}(2,493)=380.3094 \\
(\mathrm{p}=0.0000)\end{array}$ \\
\hline $\begin{array}{c}\text { ¿Cuántas horas gastas mandando o leyendo } \\
\text { mensajes en un día? (incluye SMS, WhatsApp, } \\
\text { Facebook). Típico día durante el fin de semana. }\end{array}$ & $\begin{array}{c}\text { Media }=5.1969 \\
\text { Muestra }=138\end{array}$ & $\begin{array}{c}\text { Media }=7.6289 \\
\text { Muestra }=138\end{array}$ & $\begin{array}{c}\mathrm{F}(2,493)=187.145 \\
(\mathrm{p}=0.0000)\end{array}$ \\
\hline $\begin{array}{c}\text { ¿Cuántas horas gastas mandando o leyendo } \\
\text { mensajes en un día? (incluye SMS, WhatsApp, } \\
\text { Facebook). Típico día durante las vacaciones. }\end{array}$ & $\begin{array}{c}\text { Media }=6.7017 \\
\text { Muestra }=138\end{array}$ & $\begin{array}{c}\text { Media }=8.8463 \\
\text { Muestra }=138\end{array}$ & $\begin{array}{c}\mathrm{F}(2,493)=184.664 \\
(\mathrm{p}=0.0000)\end{array}$ \\
\hline
\end{tabular}

Tamaño de la muestra 707. Nivel de confianza de $95 \%$.

5) Al igual que lo manifestó el grupo de jóvenes participantes en las encuestas focales, la aplicación que más prefieren para comunicarse con su familia y amigos es a través del WhatsApp (' $y$ el uso del audioteléfono). Más de $80 \%$ de los jóvenes señaló que es muy importante la red social para comunicarse con su familia y amigos. Por otra parte, hablar por teléfono (audio) sigue siendo el medio preferido por los jóvenes cuando se trata de la familia (figura 2).

Respecto del género, puede decirse que los varones son más precisos en decir que utilizan el audio y el texto, a diferencia de las mujeres, que reconocen la combinación de las distintas redes sociales para comunicarse con sus amigos (tabla 4 ). 


\section{Figura 2. Aplicación y medio elegido por los jóvenes para comunicarse con su familia y amigos}

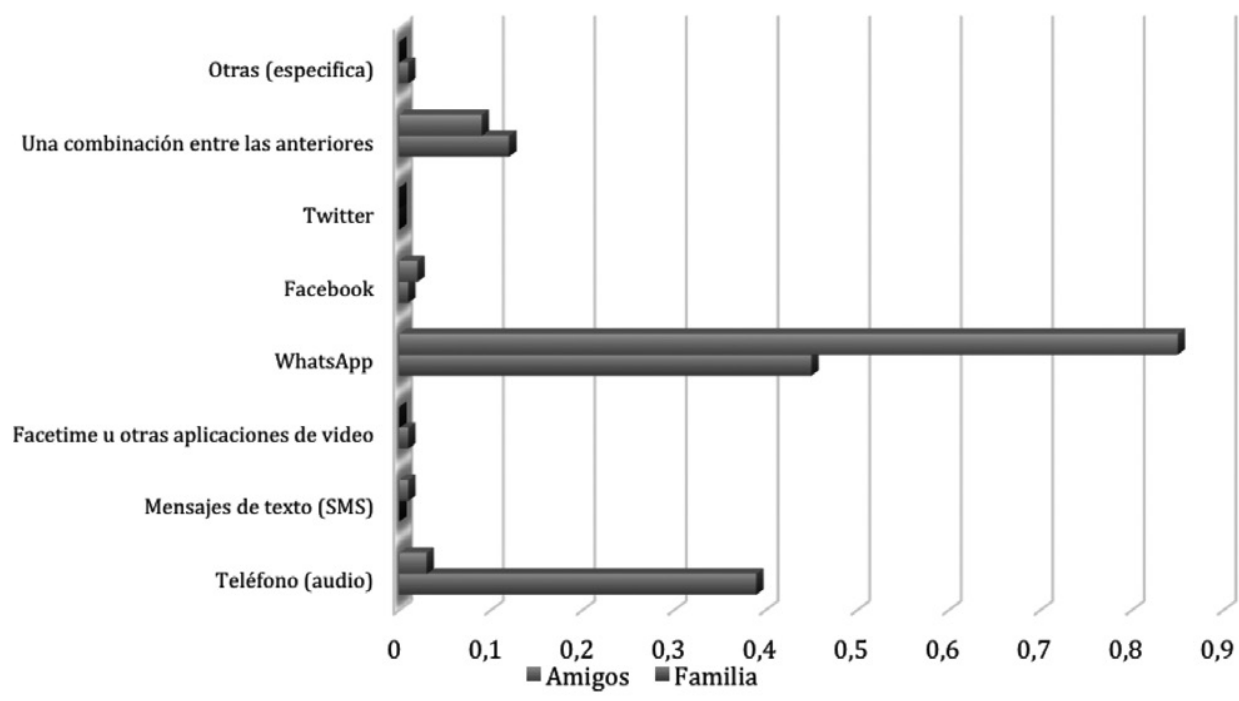

Tamaño de la muestra 707

Tabla 4. Tipo de aplicación preferida por los jóvenes mexicanos para comunicarse con sus amigos

\begin{tabular}{|l|c|c|}
\hline \multirow{2}{*}{} & \multicolumn{2}{|c|}{ Género } \\
\cline { 2 - 3 } & Masculino & Femenino \\
\hline Teléfono (voz) & 4.35 & 2.54 \\
\hline Mensajes de texto & 1.45 & 0.32 \\
\hline FaceTime u otras aplicaciones de video & 0 & 0 \\
\hline WhatsApp & 86.23 & 85.71 \\
\hline Facebook & 2.17 & 0.63 \\
\hline Twitter & 0 & 0 \\
\hline Combinación de los anteriores & 5.8 & 10.16 \\
\hline Otros & 0 & 0.63 \\
\hline
\end{tabular}

i $2=298.912$, p-value $=0.0000$. Tamaño de la muestra 707 . Nivel de confianza de $95 \%$.

6) El $93 \%$ de los jóvenes identifica como muy importantes usar sus smartphones para mantenerse en contacto con la familia y los amigos, $78 \%$ para tener acceso a noticias e información y $67 \%$ para mayor privacidad en la comunicación. Sin embargo, solo 52.83 \% conside- 
ra como muy y algo importante este medio para sentirse seguros. El $78 \%$ considera este dispositivo como muy y algo importante para tener acceso a información y noticias (tabla 5).

Tabla 5. Valoración de los jóvenes mexicanos respecto de la importancia que tiene su smartphone

\begin{tabular}{|l|c|c|c|c|c|}
\hline & $\begin{array}{c}\text { Muy } \\
\text { importante } \\
(\mathbf{1})(\mathbf{\%})\end{array}$ & $\begin{array}{c}\text { Algo } \\
\text { importante } \\
(\mathbf{2})(\mathbf{\%})\end{array}$ & $\begin{array}{c}\text { Neutral } \\
(\mathbf{3})(\mathbf{\%})\end{array}$ & $\begin{array}{c}\text { No muy } \\
\text { importante } \\
(\mathbf{4}) \mathbf{( \% )}\end{array}$ & $\begin{array}{c}\text { Nada } \\
\text { importante } \\
(\mathbf{5}) \mathbf{\%})\end{array}$ \\
\hline Mantenerme en contacto con familia y amigos & 67 & 26 & 6 & 0 & 0 \\
\hline Mayor privacidad en la comunicación & 33 & 34 & 26 & 4 & 2 \\
\hline Sentirte seguro & 27 & 30 & 32 & 7 & 4 \\
\hline Mantener cierto nivel de estatus social & 7 & 19 & 38 & 18 & 19 \\
\hline Acceso a noticias e información & 36 & 42 & 18 & 3 & 1 \\
\hline Ver televisión y películas & 7 & 16 & 28 & 24 & 26 \\
\hline Pasar el tiempo jugando conectado & 4 & 5 & 20 & 24 & 48 \\
\hline Pasar el tiempo jugando desconectado & 2 & 6 & 20 & 27 & 45 \\
\hline
\end{tabular}

Tamaño de la muestra 707.

Es baja la valoración que le dan los jóvenes a su celular para su estatus social. Solo 26 \% lo considera como muy y algo importante. Es decir, para casi $74 \%$ de la población encuestada no está considerado como reflejo de estatus social. Por otra parte, $23 \%$ de los jóvenes considera como muy y algo importante para ver televisión y noticias. De los juegos en línea y fuera de línea, el porcentaje disminuye. Tan solo $9 \%$ es considerado para juegos en línea y $8 \%$ para los juegos fuera de línea. Esta generación no valora como algo significativo el smartphone para jugar ni tampoco para escuchar radio.

8) Los jóvenes prefieren como primera opción el smartphone comparado con otros dispositivos móviles para escuchar su música favorita, bajar música de internet, navegar en internet y acceder a sus sitios favoritos, ver noticias y contenidos informativos, compartir información con los amigos, ver videos en YouTube, videojuegos, así como leer y enviar correos electrónicos.

También puede decirse que el celular es el medio preferido por los jóvenes comparado con otros medios, como la tableta, el MP3 player, la 
laptop, la computadora de escritorio y la radio. Solo la laptop fue considerada como el medio elegido número uno para ver películas o programas de televisión, comparado al smartphone y el resto de los medios.

Por último, existe una relación directa entre el género de la persona y la importancia que le dan al uso de los diferentes medios electrónicos. Por ejemplo, $58 \%$ de las personas clasificó el celular como el medio favorito para escuchar música. Pero para las mujeres es más significativo en comparación a la valoración que le dan los hombres, a diferencia de lo que sucede con la tableta. En este sentido, puede interpretarse que para las mujeres el uso y la gratificación que les da el smartphone es más significativo e importante que para los varones en los siguientes aspectos: para escuchar su música preferida, navegar en internet, ver películas o programas de televisión, compartir información con sus amigos, ver videos en YouTube, videojuegos, así como para leer y enviar correos electrónicos (tabla 6).

\section{Tabla 6. Preferencias de los jóvenes mexicanos para realizar actividades específicas en los dispósitivos móviles}

\begin{tabular}{|c|c|c|c|c|}
\hline & & & \multicolumn{2}{|c|}{ Género } \\
\hline & Frecuencia & $\%$ & Masculino (\%) & Femenino (\%) \\
\hline \multicolumn{5}{|c|}{ Para escuchar mi música favorita } \\
\hline 1. Smartphone & 288 & 58.0 & 58.7 & 65.4 \\
\hline 2. Tableta & 98 & 19.7 & 26.1 & 19.7 \\
\hline 3. MP3 player & 35 & 7.0 & 8.7 & 7.3 \\
\hline 4. Laptop & 21 & 4.2 & 4.4 & 4.1 \\
\hline 5. Computadora de escritorio & 12 & 2.4 & 1.5 & 3.2 \\
\hline 6. Radio & 2 & 0.4 & 0.7 & 0.3 \\
\hline \multicolumn{5}{|c|}{ i 2 con 12 grados de libertad $=466.8198(\mathrm{p}=0.0000)$} \\
\hline \multicolumn{5}{|c|}{ Para navegar en internet $y$ acceder a mis sitios favoritos } \\
\hline 1. Smartphone & 232 & 46.7 & 44.2 & 54.3 \\
\hline 2. Tableta & 126 & 25.4 & 31.9 & 26.0 \\
\hline 3. MP3 player & 72 & 14.5 & 20.3 & 13.3 \\
\hline 4. Laptop & 23 & 4.6 & 2.9 & 5.7 \\
\hline 5. Computadora de escritorio & 12 & 0.6 & 0.7 & 0.6 \\
\hline 6. Radio & 0 & 0.0 & 0.0 & 0.0 \\
\hline
\end{tabular}




\begin{tabular}{|c|c|c|c|c|}
\hline & & & \multicolumn{2}{|c|}{ Género } \\
\hline & Frecuencia & $\%$ & Masculino (\%) & Femenino (\%) \\
\hline \multicolumn{5}{|c|}{ Para ver noticias y contenidos informativos } \\
\hline 1. Smartphone & 317 & 63.8 & 66.7 & 71.1 \\
\hline 2. Tableta & 73 & 14.7 & 18.1 & 15.2 \\
\hline 3. MP3 player & 38 & 7.7 & 8.7 & 7.6 \\
\hline 4. Laptop & 23 & 4.6 & 3.6 & 5.7 \\
\hline 5. Computadora de escritorio & 4 & 0.8 & 2.2 & 0.3 \\
\hline 6. Radio & 1 & 0.2 & 0.7 & 0.0 \\
\hline \multicolumn{5}{|c|}{ i 2 con 12 grados de libertad $=469.6698(\mathrm{p}=0.0000)$} \\
\hline \multicolumn{5}{|c|}{ Para ver videos de YouTube o de servicios similares } \\
\hline 1. Smartphone & 246 & 49.5 & 49.3 & 56.2 \\
\hline 2. Tableta & 113 & 22.7 & 30.4 & 21.9 \\
\hline 3. MP3 player & 74 & 14.9 & 15.2 & 16.8 \\
\hline 4. Laptop & 19 & 3.8 & 2.9 & 4.8 \\
\hline 5. Computadora de escritorio & 4 & 0.8 & 2.2 & 0.3 \\
\hline 6. Radio & 0 & 0.0 & 0.0 & 0.0 \\
\hline \multicolumn{5}{|c|}{ i 2 con 12 grados de libertad $=469.4567(\mathrm{p}=0.0000)$} \\
\hline \multicolumn{5}{|c|}{ Para leer y mandar correos } \\
\hline 1. Smartphone & 224 & 45.1 & 43.5 & 52.1 \\
\hline 2. Tableta & 128 & 25.8 & 28.3 & 27.3 \\
\hline 3. MP3 player & 65 & 13.1 & 15.2 & 14.0 \\
\hline 4. Laptop & 36 & 7.2 & 11.6 & 6.4 \\
\hline 5. Computadora de escritorio & 3 & 0.6 & 1.5 & 0.3 \\
\hline 6. Radio & 0 & 0.0 & 0.0 & 0.0 \\
\hline \multicolumn{5}{|c|}{ i 2 con 12 grados de libertad $=468.0926(p=0.0000)$} \\
\hline \multicolumn{5}{|c|}{ Para bajar mi música de internet } \\
\hline 1. Smartphone & 169 & 34.0 & 38.4 & 36.8 \\
\hline 2. Tableta & 125 & 25.2 & 26.8 & 27.3 \\
\hline 3. MP3 player & 87 & 17.5 & 22.5 & 17.8 \\
\hline 4. Laptop & 52 & 10.5 & 7.4 & 12.1 \\
\hline 5. Computadora de escritorio & 19 & 3.8 & 2.2 & 5.1 \\
\hline 6. Radio & 4 & 0.8 & 0.7 & 1.0 \\
\hline
\end{tabular}




\begin{tabular}{|c|c|c|c|c|}
\hline & \multirow[b]{2}{*}{ Frecuencia } & \multirow[b]{2}{*}{$\%$} & \multicolumn{2}{|c|}{ Género } \\
\hline & & & Masculino (\%) & Femenino (\%) \\
\hline \multicolumn{5}{|c|}{ Para ver películas o programas de televisión } \\
\hline 1. Smartphone & 291 & 58.6 & 58.0 & 66.4 \\
\hline 2. Tableta & 104 & 20.9 & 24.6 & 21.9 \\
\hline 3. MP3 player & 22 & 4.4 & 6.5 & 4.1 \\
\hline 4. Laptop & 29 & 5.8 & 8.7 & 5.4 \\
\hline 5. Computadora de escritorio & 10 & 2.0 & 2.2 & 2.2 \\
\hline 6. Radio & 0 & 0.0 & 0.0 & 0.0 \\
\hline \multicolumn{5}{|c|}{ i 2 con 12 grados de libertad $=464.6387(\mathrm{p}=0.0000)$} \\
\hline \multicolumn{5}{|c|}{ Para compartir información con amigos } \\
\hline 1. Smartphone & 410 & 82.5 & 82.6 & 93.3 \\
\hline 2. Tableta & 30 & 6.0 & 11.6 & 4.1 \\
\hline 3. MP3 player & 6 & 1.2 & 2.2 & 1.0 \\
\hline 4. Laptop & 9 & 1.8 & 3.6 & 1.3 \\
\hline 5. Computadora de escritorio & 1 & 0.2 & 0.0 & 0.3 \\
\hline 6. Radio & 0 & 0.0 & 0.0 & 0.0 \\
\hline \multicolumn{5}{|c|}{ i 2 con 12 grados de libertad $=475.1599(\mathrm{p}=0.0000)$} \\
\hline \multicolumn{5}{|c|}{ Para video juegos } \\
\hline 1. Smartphone & 223 & 44.9 & 35.5 & 55.2 \\
\hline 2. Tableta & 86 & 17.3 & 17.4 & 19.4 \\
\hline 3. MP3 player & 66 & 13.3 & 16.7 & 13.3 \\
\hline 4. Laptop & 60 & 1 & 23.9 & 8.3 \\
\hline 5. Computadora de escritorio & 20 & 4.0 & 6.5 & 3.5 \\
\hline 6. Radio & 1 & 0.2 & 0.0 & 0.3 \\
\hline
\end{tabular}

Tamaño de la muestra 707. Nivel de confianza $95 \%$.

\section{Conclusiones}

Los jóvenes hispanos en México reconocen que el smartphone ha puesto al alcance aplicaciones que satisfacen diversas necesidades de información y entretenimiento. Este estudio muestra que a tal población de América Latina no le gusta recibir publicidad y realizar operaciones comerciales a través de su smartphone. A pesar de que este segmento de la población tiene múltiples accesos a redes sociales para comunicarse, el medio elegido de contacto con familia y amigos es el WhatsApp. Por otra parte, este dispositivo móvil se ha convertido también en un facilitador para conectarse a otros medios, como la televisión inteligente Smart TV. 
Por otra parte, la investigación muestra que el teléfono inteligente se ha convertido en el medio preferido de los jóvenes universitarios respecto de otros medios para escuchar su música preferida, navegar en internet, ver contenidos de información y de noticias, compartir información, mirar videos de YouTube, así como leer y mandar correos electrónicos. Por último, puede afirmarse que existe diferencia entre la marca de smartphone y los diferentes usos y gratificaciones según el género de la persona y el nivel de ingresos en México.

\section{Limitaciones y futuras líneas de investigación}

En esta investigación, se pone de manifiesto que los jóvenes utilizan su teléfono inteligente también para acceder a información relevante de interés noticioso. Por otra parte, manifiestan que este medio es importante para compartir información con sus amigos. Sin embargo, el estudio no presenta un comparativo respecto de la valoración y los usos que hacen del contenido informativo que presentan las diversas empresas de comunicación. Otro aspecto relevante para futuras investigaciones sería ver cómo evalúan los jóvenes universitarios los diversos esfuerzos institucionales que se hacen para comunicarse con ellos a través de las redes sociales u otros medios digitales adaptados a estos dispositivos móviles. La valoración que puedan hacer tales jóvenes es importante para identificar en qué medida las estrategias utilizadas por las diversas instituciones son adecuadas para comunicarse e interactuar con este público. Finalmente, el tema de la confianza en las instituciones mexicanas es un asunto que debería evaluarse con este tipo de estudios. Llama la atención que tales jóvenes continúan desconfiando de la publicidad y no llevan a cabo operaciones comerciales por esta razón. Por otra parte, aunque tienen acceso a contenidos informativos, llama la atención que para ellos es irrelevante la información que les llega. Este es un tema que aún prevalece como parte de la cultura mexicana y que debería analizarse el por qué. 


\section{Referencias}

Albarran, A. B. (ed.) (2013). The social media industries. Routledge: Nueva York.

Albarran, A. B. y Hutton, B. (2009). Young latinos use of mobile phones: a cross-cultural study. Revista de Comunicación, 8, 95-108.

Ampici (2015). 11 estudio sobre los hábitos de los usuarios de internet en México 2015. Recuperado el 22 de octubre de 2015 de https:// www.amipci.org.mx/images/AMIPCI_HABITOS_DEL_INTERNAUTA_MEXICANO_2015.pdf

Campbell, S. (2007). A cross-cultural comparison of perceptions and uses of mobile telephony. New Media \& Society, 9(2), 343-363.

ComScore (2015). 2015 Digital Future in Focus Reports. Recuperado el 22 de octubre de 2015 de http: / / www.comscore.com/Insights/Presentations-and-Whitepapers/2015/2015-Global-Digital-Future-in-Focus

Darcin, A., Noyan, C., Nurmedov, S., Yilmaz, O. y Dilbaz, N. (2015). Smartphone addiction in relation with social anxiety and loneliness among university students in Turkey. European Psychiatry, 30, 505.

Díaz, E. y Marrero, L. (2011). Nuevas fronteras para la participación en red: ciberperiodismo y telefonía móvil en América Latina. Telos: Cuadernos de Comunicación e Innovación, 87, 35-46.

Domínguez, F. y López, R. (2015). Uso de las redes sociales digitales entre los jóvenes universitarios en México: hacia la construcción de un estado del conocimiento (2004-2014). Revista de Comunicación, 14, 48-69.

Elizondo, A., Bernal, J. y Montoya, M. (2010). Desarrollo de habilidades cognitivas con aprendizaje móvil: un estudio de casos. Comunicar: Revista Científica Iberoamericana de Comunicación y Educación, 34, 201-209. 
Herrera, M. (2009). Disponibilidad, uso y apropiación de las tecnologías por estudiantes universitarios en México: perspectivas para una incorporación innovadora. Revista Iberoamericana de Educación, 48(6), 4.

García-De-Torres, E., Yezers'ka, L., Rost, A., Calderín, M., Edo, C., Rojano, M., y Corredoira, L. (2011). Uso de Twiter y Facebook por los medios iberoamericanos. El Profesional de la Información, 20(6), 611-620. doi:10.3145/epi.2011.nov.02

Inegi (2014). Estadísticas sobre disponibilidad y uso de tecnología de información y comunicaciones en los hogares, 2013. Recuperado el 22 de octubre de 2015 de http://www.inegi.org.mx/prod_serv/ contenidos/espanol/bvinegi/productos/metodologias/MODUTIH/MODUTIH2013/MODUTIH2013.pdf

Joo, J. y Sang, Y. (2013). Exploring Koreans' smartphone usage: An integrated model of the technology acceptance model and uses and gratifications theory. Computers in Human Behavior, 29(6), 25122518. doi:10.1016/j.chb.2013.06.002.

Katz, E., Blumler, J. G. y Gurevitch, M. (1973). Uses and gratifications research. The Public Opinion Quarterly, 37(4), 509-523.

Qiaolei, J., Lin, T. y Yi-Hsuan, C. (2015). Sociable people beware? Investigating smartphone versus non smartphone dependency symptoms among young singaporeans. Social Behavior \& Personality: An International Journal, 43(7), 1209-1216. doi:10.2224/ sbp.2015.43.7.1209

Ruggiero, T. E. (2000). Uses and gratifications theory in the 21 st century. Mass Communication \& Society, 3(1), 3-37.

Van Weezel, A. y Benavides, C. (2009). Uso de teléfonos móviles por los jóvenes. Cuadernos de Información, 5, 5-14.

Yelton, A. (2012). Who are smartphone users? Library Technology Reports, $48(1), 5-8$. 